\title{
Optimal and Efficient Energy Utilization Using the Dual Mode Energy Driven Routing Protocol for Wireless Planetary Exploration
}

\author{
S.Saravana Kumar, Neethu Prabhakaran.P \\ Assistant Professor, PG Student, Dept of CSE SVS College of Engineering, Coimbatore, Tamilnadu, India.
}

\begin{abstract}
Wireless Sensor Network is the sensing network which is most useful for detecting the environmental conditions such as pressure, temperature, density. Different autonomous sensors are contained in the Wireless Sensor Network; each sensor node is sensing and detecting the environmental conditions. Planetary Exploration is the process of searching the information about the planets and its physical and chemical and biological characteristics of each and every part of the planetary system. Adhoc On demand Distance Vector routing protocol is commonly used protocol for planetary exploration in the wireless sensor network. It has several disadvantages like energy efficiency problem, unnecessary bandwidth consumption, connection setup delay is lower. To overcome all the disadvantages of AODV protocol, the Dual Mode Energy Driven Routing Protocol is proposed. DMED protocol is mainly contains two modes, the day mode and the night mode. The day mode is the charging state of the solar battery in the planetary sensor system and night mode is the sleeping state of the solar battery. Main objective of this paper is to implement a dual mode energy driven routing protocol for optimal and efficient energy utilization and improving the energy efficiency and energy balancing in the wireless sensor network for planetary exploration.
\end{abstract}

Keywords: Planetary Exploration, Dual Mode Energy Driven Routing protocol, Wireless Sensor Network.

\section{Introduction}

Energy Efficiency problem is the challenging problem in many networks. All the satellites system and spacecraft system needs to maintain the energy and balancing the energy in the optimal and efficient way. Planetary condition is challenged by different environmental conditions such as energy and density and special temperature. To detect the environmental conditions wireless sensor network is to be used. In the wireless sensor network contains the different spatially distributed autonomous sensor nodes and every sensor node is sensing and detecting the environmental conditions such as pressure, sound, temperature etc.

DMED protocol is specially developed for the special environmental conditions and at the same time DMED is used for improving the energy efficiency. Optimal and efficient energy utilization is the another important criteria of this protocol. DMED protocol uses the energy in the optimal and the efficient way.

There are different sources of energy such as solar energy, wind energy. DMED mainly uses the solar energy which is the most important and most useful renewable energy resource. Solar energy comes from the sun in the reaction of thermo nuclear fusion. There are different types of sensor nodes in the planetary surface sensor system used for gathering the solar energy. DMED improves the network life time efficiently and effectively. In the case of DMED energy utilization and energy consumption is decreased effectively with the strategy of combining the circular path and the shortest path. The routing scheme balances the energy among multiple and different paths.

Battery and the solar energy are the two different types of the energy source in the solar systems. In the case of vibrate and thermal energy are very difficult to harvest. There are several techniques to be used for the battery resistance performance with special temperature. According to the mars exploration rover, there are mainly three lands of space craft battery system. Rechargeable battery, Landers primary battery back shells thermal battery these are the most important batteries used in the space craft systems. The researchers shows that lithinum ion rechargeable battery is lighter and more compact than the conventional batteries. Lithinium ion rechargeable battery system are used in the planetary surface sensor system.

\section{Day Mode In DMED}

Solar energy is the most important and most efficient renewable energy resource. In the planetary surface sensor are the autonomous sensors and at the same time it is performed as efficient solar batteries and the day time solar batteries are to be charged. At this time find the optimal shortest path between the source node and sink node and this time route stable is the key design

The planetary surface sensor system is embedded with the highly solar powered sensor node and it is charging in the day mode in the day time and sleeping in the night mode in the night time. Wireless sensor 
network supports the DMED protocol for the planetary exploration. DMED mainly used for achieving the energy efficiency.

\section{Night Mode In DMED}

Energy as the key design point in the case of night mode. In the case of night mode energy of the nodes dropped sharply because each node in the planetary surface sensor is energy challenged. DMED maintains the node energy with the help of energy balancing. Energy balancing is the one of the most important criteria in DMED.

\section{Wsn Model Of Planetary Exploration}

WSN model of planetary exploration consist of different systems. The important systems are the satellite sensor system, Planetary surface sensor system, event management system and internet. Planetary surface sensor system is embedded different sensor nodes and these are equipped in the day mode and night mode. The network node deployment can be controlled.

The WSN model for planetary exploration system is as shown in figure 1. The networking in the planetary system processed by the self -organized way and the data is to be transferred to the data processing center in the earth. Each communication range in the planetary system is limited and the data transmission in a multi hop way. High performance is required the sink node and the energy is to be infinite.

Different systems in the WSN model of planetary exploration.

1.Satelite sensor system.

2.Planetary surface sensor system

3.Event management system

4.Internet

The planetary surface sensor system is the very important model in this paper which works in the dual mode ie battery charging mode and battery sleeping mode these modes are referred to as charging mode and sleeping mode.

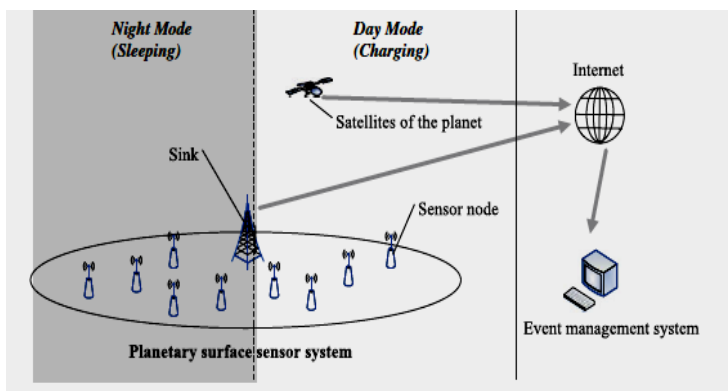

Figure 1: WSN model for planetary exploration

\section{DMED Routing Protocol}

Improving the communication quality and energy balancing in the whole network are the main objective of the DMED routing protocol. DMED protocol mainly contains different mechanisms .

1.WAR routing consultation mechanism

2.Multi-Route Request Processing mechanism

3.route checking mechanism

\section{WAR Routing Consultation Mechanism}

Key design of the WAR routing consultation mechanism is improving the communication quality and the energy balancing. In this method first to find the shortest path in the network and the energy of the each nodes in the network and next to find the remaining energy of the intermediate nodes and these are compared in the path finding process then it is checked with the later and it is less than the later the routing tableof the networking system should be updated. If the Route Request message(RREQ) and Route Reply(RREP) messages reach their destination then the minimum residual energy is to be calculated.

The Routing Consultation mechanism is between the energy of the each nodes and the hop counts. DMED uses the Weighted Average Of Reciprocal mechanism to find the finite computing ability of the sensor nodes.

Routing Consultation is the different step procedure and it is described in this section using the mathematical formulas. In the routing Consultation contains the $\mathrm{i}(\mathrm{i} \in \mathrm{N}=(2,3, \ldots \mathrm{N})$ paths and pi indicates the i path and Ei indicates the minimum nodal energy and $\mathrm{Hi}$ is the no of hop count and wi is the weight of pi. This 
routing consultation method is referred as WAR.The weight of $\mathrm{Wi}=\mathrm{pei}+\mathrm{q} 1 / \mathrm{Hi}$, where $\mathrm{p}$ and $\mathrm{q}$ denoted as the weighted coefficients and $p+q=1$ then wi=pei+(1-p)1/hi DMED finds the optimum path according to its energy and according to its hop counts and the generator ratio of $\mathrm{p}$ is described as $1 / \mathrm{Hi}+\mathrm{p}(\mathrm{Ei}-1 / \mathrm{Hi})$.In dual working mode $\mathrm{p}$ is adjusted between the working of two different modes. The value of $\mathrm{p}$ will impact the routing choice. When $\mathrm{p}=0$ then the AODV routing mechanism will be obtained. In this method AODV is the special case of DMED.

\section{Multi Route Request Processing Mechanism}

Energy is the most important factor in the DMED routing protocol and energy is involved in the path weight calculation of WAR.DMED handles the RREQ messages and the multi route request processing mechanism only effects the destination node so the original RREQ broadcast time not to be increased. The request processing mechanism ensures that there is no repeated loop among the multiple and different paths and there is no repeated node in the network

\section{Route Checking Mechanism}

DMED does not maintain the multiple paths at the same time or simultaneously. If the data transmission are large then there are several problems like the energy of the working mode sometimes will be cut down and in the case of energy consumptions the nodes will be invalid. Because of this problems a regular route checking mechanism is to be needed.

In the DMED route checking mechanism, if the no of data packets which have been successfully transferred are reached successfully to the certain value then the RREQ messages are requested to rebroadcast once and the route finding process is to be restarted. This is known as route switching principle and the DMED maintains the optimal routing effectively and efficiently.

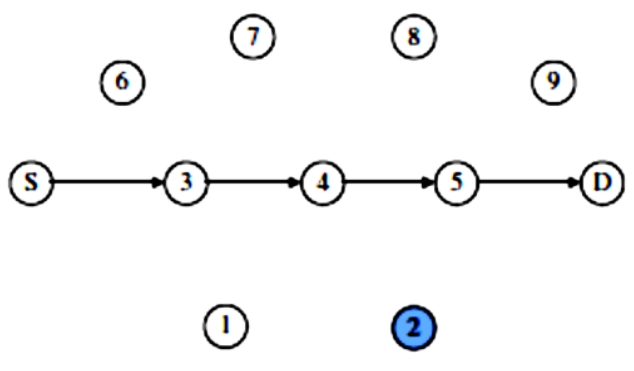

(a)

Figure 2: The regular route checking mechanism (a)The first path finding process

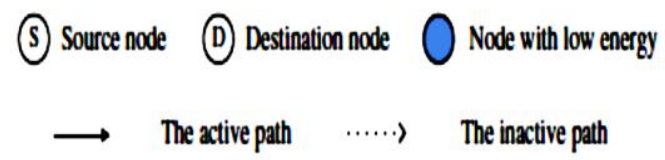

The Figure 2(a) shows the regular route checking mechanism and it first compute the path and the energy is selected after the route finding process. In the first step, to find the shortest path between the source and the destination .In the first step remaining nodes does not participate in the communication.

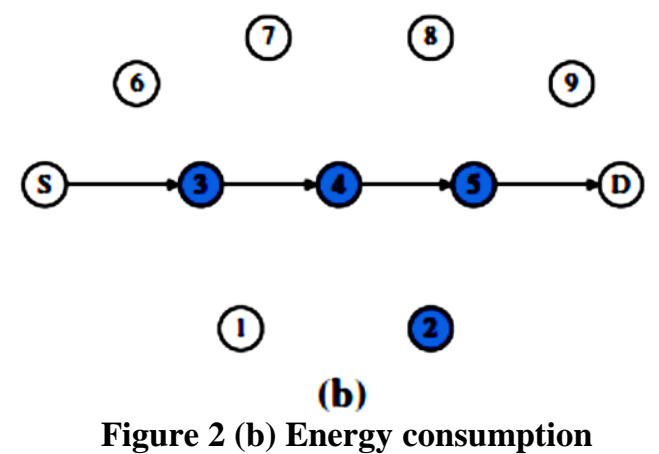


The Figure 2(b) shows the energy of the nodes, in this figure node 3,4 and 5.If they are working long time with this path the path is decreased shortly then the path is not suitable for the data transmission of the data packets.

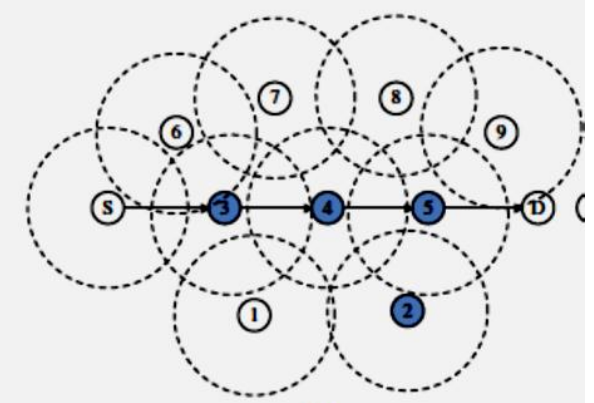

(c)

Figure 2(c) The regular broadcasting mechanism

Figure2 (c) shows the regular broadcasting mechanism and it helps to maintain the energy consumption in the different nodes. In this step DMED recalculates the value of weighted average of reciprocals and to find the optimal and suitable path and this path will be chooses as the next path.

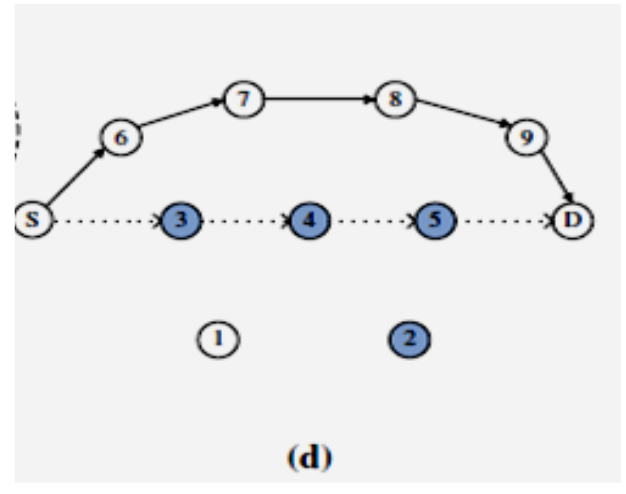

Figure 2(d) The Route Switching Process

Figure 2(d) shows the route switching process and $\mathrm{S}->6->7->8->9->\mathrm{D}$ is the path in the figure. The path which is shown in the figure is the optimal path and it is based on the route switching principle. Due to the Route checking process, DMED maintains very efficient and nice energy balancing performance.

\section{Dual Working Mode}

In both the day mode and the night mode the energy of the nodes is consumed persistently even if the nodes does not participates the communication. The examples are given below.

The Procedures Of Sending

Receiving and Forwarding the data need to consume the energy

Dual working mode is developed for adjusting the route finding process. The rechargeable solar batteries are charging in the day mode and sleeping in the night mode then the association of energy driven routing mechanism can balance the energy effectively and efficiently. At this time route finding process is to find the shortest path and the communication quality can be improved by the appropriate $p$ value in the WAR function 


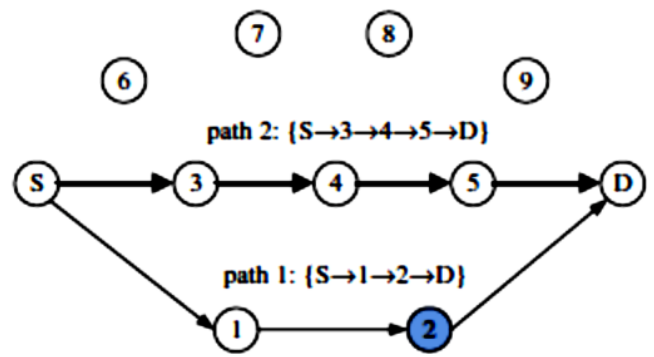

(a)

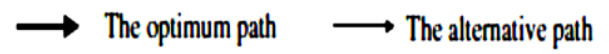

Figure 3(a): The path finding process under the day mode

Figure 3 describes the path finding process

under the day mode .Node 2 is the lower energy node in the figure and the path1 $\{\mathrm{S}->1->2->\mathrm{D}\}$ can be the alternative path because it has the minimum hop counts.

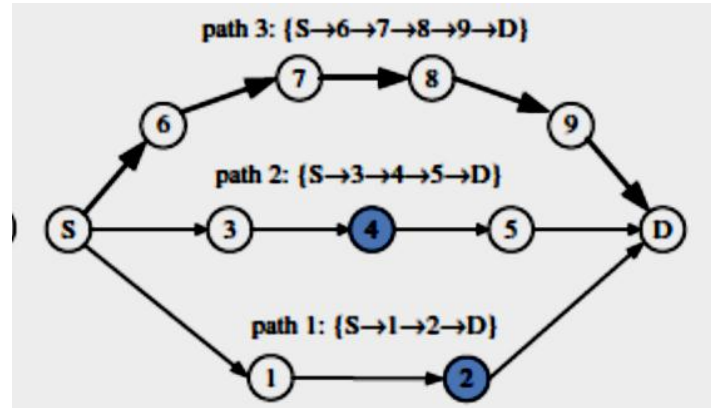

(b)

Figure 3(b)The path finding process under the night mode

Figure 3(b) shows the path finding process under the night mode. Under the night mode there is no solar energy for battery charging so the energy of each node is dropped sharply. To avoid this problem the route finding process under the night mode is to distribute the traffic burden into more nodes. In this the path which has the sufficient energy is inclined to be chosen.

The energy of the node 2 is not enough and path 3 is to be find the optimal one according to the WAR routing consultation mechanism. S->6->7->8->9->D is the optimal path in the figure 4(b). When dealing with the three route request (RREQ) messages under the night mode, the route is to be switched one or two times to find the optimal one with enough energy and this real time routing process does not affect the data transmission process.

\section{Experiments and Analysis Methods}

In this paper the performance of the AODV is compared with the DMED and the analysis and the experiments shown that the DMED routing protocol is the most useful and most effective routing protocol in the conventional wireless sensor networks and it helps to improve the energy efficiency and to maintain the energy balancing in the wireless sensor networks.

The Simulation settings and the simulation parameters are described in the table1.The experimental Tool is NS-2 it is the most important simulation tool which is universally accepted multiprotocol simulation software and it is developed by the DARPA and it is supported by the Virtual InterNetTestBed.The radio propagation model we use two ray ground and it is commonly used in the mobile scenarios. The motivation of the networking simulation is to learn the fundamentals of evaluating the network performance and to analyse the overall network performance. To implement the dual mode energy driven routing protocol Ns2 simulation is to be used and Ns2 simulation is the most fundamental type of discrete event simulation.Ns2 provides the substantial and essential support for the simulation of the TCP protocol and different routing process 
Table 1 The Simulation Settings

\begin{tabular}{|l|l|}
\hline \multicolumn{1}{|c|}{ Simulation parameter } & \multicolumn{1}{c|}{ value } \\
\hline Simulation & NS-2(version 2.35) \\
\hline Channel & Wireless \\
\hline MAC layer Protocol & 802.11 \\
\hline Network range & $1.500 \mathrm{~m}^{*} 1.500 \mathrm{~m}$ \\
\hline Number of nodes & 150 \\
\hline Initial energy of each node & $15 \mathrm{nj}$ \\
\hline Maximal transmission range & $250 \mathrm{~m}$ \\
\hline Radio propagation model & Two ray ground \\
\hline Maximal packet rate & 60 \\
\hline Packet size & 1024 byte \\
\hline Traffic type & CBR(constant bytes rate) \\
\hline Mobility model & Random way point \\
\hline
\end{tabular}

There different types of simulation tools is to be used.NS-2 and NS-3 simulation are most important simulation tools.Simulation is the process of simulating the real word process.Space simulation and fight simulation are the examples of the networking simulations.Analytical and numerical techniques are used in the simulation

\section{Validation of Energy Balancing}

The energy balancing considerations of the AODV is shown in the figure.In the experiments the simulation scene is taken as 500 then the day mode and night mode occupy 250 respectively

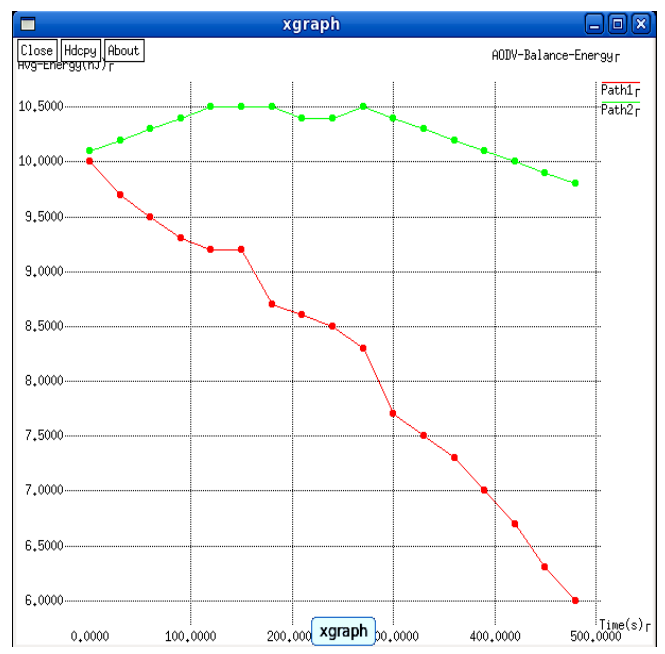

Figure 4:Energy Balancing Performance of AODV in 500s

In this rechargeable battery factor $=0.02$ and the discount rate $r=0.02$ then the energy balancing considerations of DMED and AODV is compared and to find the better performance. 


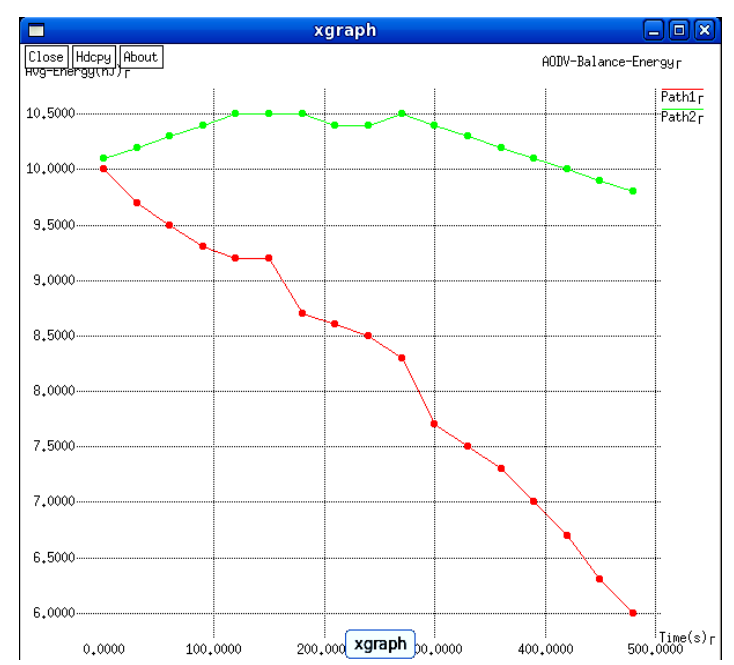

Figure 5:Energy Balancing Performance of DMED in 500s

The energy balancing performance in the DMED is shown in the figure 5.The graph shows the overall energy balancing performance in 500s.In the comparison of AODV and DMED ,DMED shows that it is more useful and efficient energy balancing performance and AODV is less energy balancing considerations comparing the DMED.

\section{Packet Delivery Ratio}

The packet delivery ratio of the DMED and AODV is to be shown in the following figure.

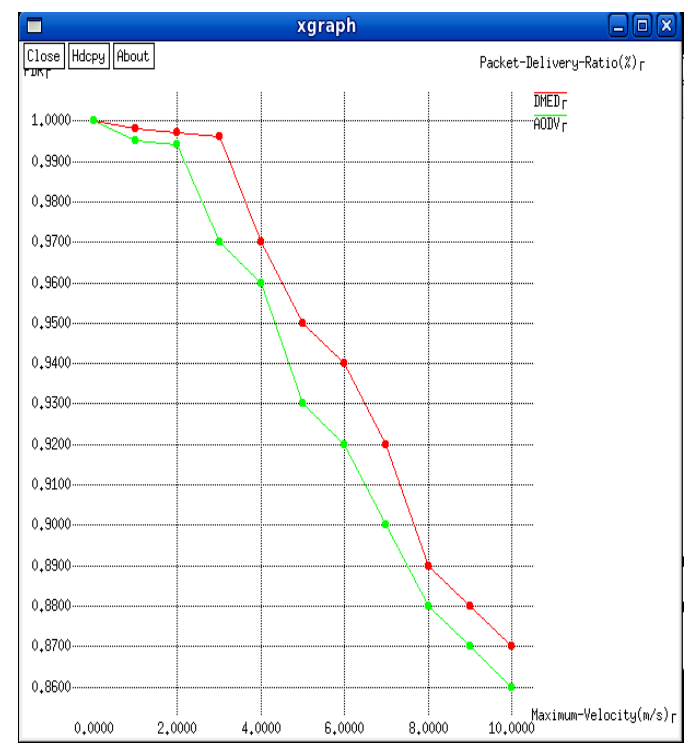

Figure 6:Performance comparison of packet delivery ratio

In the figure the two curves are downwards because of the node movement and need to consume the energy. The greater velocity, the faster energy drop, and then the different serious packet delivery ratio obtained 


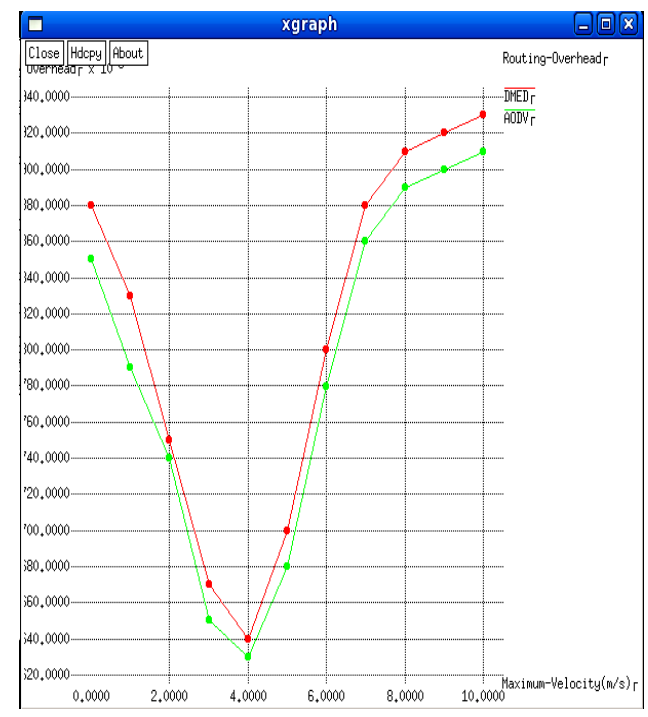

Figure 7: Performance comparison of routing overhead

The Figure 7 shows the overall performance comparision of routing head of the DMED and AODV.The red line indicates the DMED routing protocol overhead and the Green line indicates the routing overhead of the AODV and the different experiments and analysis shown that the DMED routing protocol gives the better performance and the route stability .

\section{Packet Delivery Ratio}

The packet delivery ratio of the DMED and AODV is to be shown in the following figure.

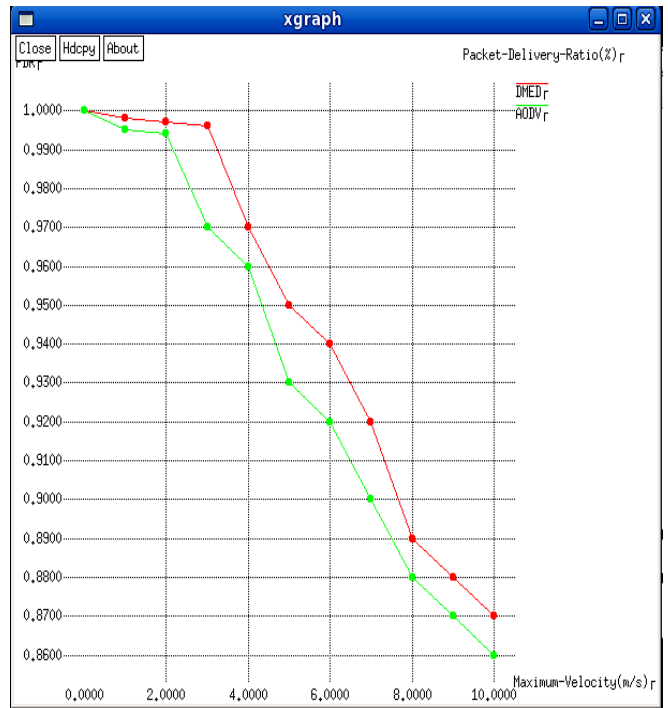

Figure 8:Performance comparison of packet delivery ratio

The packet delivery ratio is the ratio between the packets and its delivery. Packet delivery ratio is referred to as the ratio of the number of the successfully delivered data packet to the destination. To evaluate the protocol performance packet delivery ratio is to be used. If the packet delivery ratio is the greater value then it is the better performance of the routing protocol.

In the figure the two curves are downwards because of the node movement and need to consume the energy. The greater velocity, the faster energy drop, and then the different serious packet delivery ratio obtained. 


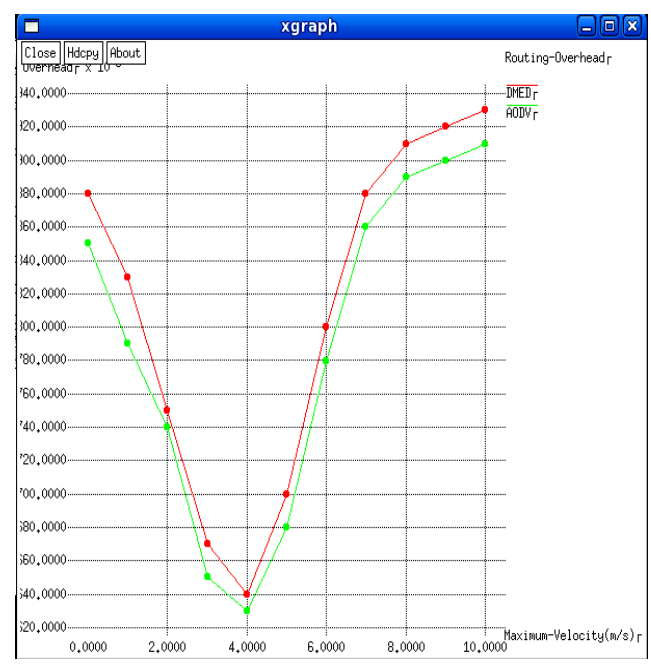

Figure 9:Performance comparison of routing overhead

The Figure 9 shows the overall performance comparison of routing head of the DMED and AODV.The red line indicates the DMED routing protocol overhead and the Green line indicates the routing overhead of the AODV and the different experiments and analysis shown that the DMED routing protocol gives the better performance and the route stability .

\section{Simulation Results And Conclusion}

According to the simulation results the Dual mode energy driven routing protocol is most usable and efficient than the adhoc on demand distance vector routing protocol and DMED is to be used for improving the communication quality and energy efficiency and at the same time performs the energy balancing of overall network. Simulation is the one of the most useful technique for the analysis and the evaluation of the networking performance and it is the model of the real world object or real time system. There are different types of simulators are to be used.Ns2 and Ns3 are the most important discrete event simulators targeted at the networking research areas.

Simulation helps to find the bugs in the design in advance and to evaluate the design alternatives in the networking research areas and to evaluate the complex functions. Space simulation and fight simulation are the examples of the networking simulations.

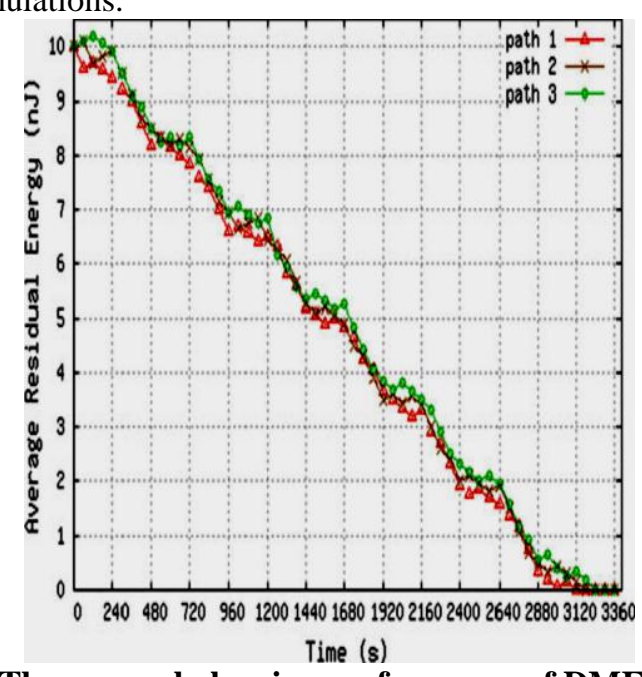

Figure:10 The energy balancing performance of DMED in 3.360s

This paper is presented for improving the energy efficiency and performing the energy balancing using the dual mode energy driven routing protocol in the wireless sensor network for the planetary exploration. there are mainly two modes are to be used the day mode and the night mode. The day mode is the charging state and the night mode is the sleeping state. 
Routing protocols are used for finding the routes or optimal path between the different nodes. Planetary exploration is the process of searching the discovery of the planets in formations and to collect its biological and physical and chemical characteristical informations.

\section{References}

[1] Dargie,W(2011)," dynamic power management in wireless snesornetworks:state of the art “ IEEE sensors Journal 12(5),1518-1528.

[2] Dubois,p..Botteron,c.,Mitev.,et al(2009) "Adhoc wireless sensor networks for exploration of solar system bodies" ,Acta Astronautica,64(5-6),626-643

[3] Durga Prasad, K., \&Murty, S. V. S. (2011)."Wireless sensor networks a potential tool to probe for water on Moon. Advances in Space Research", 48(3), 601-612

[4] Marina, M. K., \& Das, S. R. (2006). “Ad hoc on-demand multipath distance vector routing. Wireless Communications and Mobile Computing", 6, 969-988.

[5] Zarifzadeh, S., Nayyeri, A., Yazdani, N., et al. (2009). "Joint range assignment and routing to conserveenergy in wireless ad hoc networks".Computer Networks, 53(11), 1812-1829.

[6] Vidhyapriya, R., \&Vanathi, P. T. (2007)."Energy aware routing for wireless sensor networks".InProceedings of international conference on signal processing, communications and networking (pp. 545-550). 